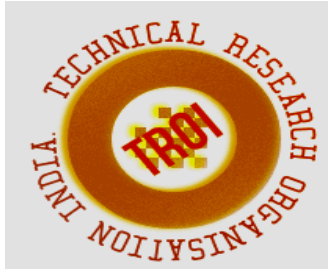

\title{
DESIGN AND DEVELOPMENT OF SINGLE AXIS SOLAR TRACKER OF SMART MONITORING SYSTEM USING IOT
}

\author{
${ }^{1}$ Kori Veeresha, ${ }^{2}$ Swetha S.R, ${ }^{3}$ Vijayalakshmi.S, ${ }^{4}$ Dr.N.Lakshmipathy, \\ ${ }^{1,2,3}$ Eight Semester UG Scholar Department of Electrical and Electronics Engineering, Dr.TTIT, \\ Oorgaum, K.G.F - 563120, India \\ ${ }^{4}$ Professor, HOD, Department of Electrical \& Electronics Engineering,Dr.TTIT, Oorgaum, K.G.F - \\ 563120, India \\ E-mail I.D: ${ }^{1}$ veeranagouda048@gmail.com \\ 2srswetharamappa@gmail.com, ${ }^{3}$ vijudevi24@gmail.com, ${ }^{4}$ nlplakshmipathy@gmail.com
}

\begin{abstract}
Solar Energy has been the power supply of choice for Industrial applications, where power is required at remote locations. Solar energy great benefit is that, it is highly reliable and requires little maintenance so it's ideal in places that are hard to get to.Electric power from solar panels is derived by converting solar power from the rays of the sun into electrical current in the solar cells. The main challenge is to maximize the capture of the rays of the sun upon the solar panels, which in turn maximizes the output of electricity.A practical way of achieving this is by positioning the panels such that the rays of the sun fall perpendicularly on the solar panels by tracking the movement of the sun. This can be achieved by means of using a solar panel mount which tracks the movement of the sun throughout the day. A single-axis solar tracker follows the movement of the sun from east to west by rotating the structure along the vertical axis. The solar panels are usually tilted at a fixed angle corresponding to the latitude of the location. Our aim is to design and fabricate the system, which will automatically track the sun's position and accordingly change the direction of the solar panel to get the maximum output from the solar cell. A solar charge controller is also designed to charge the battery.

Keywords: Payload, H-bridge, Solar Panel, PSRAM, Parallel Inverter Circuit, French Physicist Gaston Planteand.
\end{abstract}

\section{I.INTRODUCTION}

Solar Energy has been the power supply of choice for Industrial applications, where power is required at remote locations. Solar energy is also frequently used on transportation signaling e.g. Lighthouses and increasingly in road traffic warning signals. Solar energy great benefit is that, it is highly reliable and requires little maintenance so it's ideal in places that are hard to get to. Electric power from solar panels is derived by converting solar power from the rays of the sun into electrical current in the solar cells. The main challenge is to maximize the capture of the rays of the sun upon the solar panels, which in turn maximizes the output of electricity. A practical way of achieving this is by positioning the panels such that the rays of the sun fall perpendicularly on the solar panels by tracking the movement of the sun. This can be achieved by means of using a solar panel mount which tracks the movement of the sun throughout the day. A single-axis solar tracker follows the movement of the sun from east to west by rotating the structure along the vertical axis. The solar panels are usually tilted at a fixed angle corresponding to the latitude of the location. Our aim is to design and fabricate the system, which will automatically track the sun's position and accordingly change the direction of the solar panel to get the maximum output from the solar cell. A solar charge controller is also designed to charge the battery. A solar tracker is a device that orients a payload toward the sun. Payloads can be photovoltaic panels, reflectors, lenses or other optical devices. In flat-panel 
photovoltaic (PV)applications, trackers are used to minimize the angle of incidence between the incoming sunlight and a photovoltaic panel. This increases the amount of energy produced from a fixed amount of installed power generating capacity. Solar panels are effective in means of collecting solar energy, their efficiency in doing so is directly related to their angle of exposure with the sun. Because photovoltaic cells get the most energy from direct facing the sun, a stationary panel collects less sunlight than compared with that follows the sun across the sky. The problem of this project addresses is the inefficiency associated with fixed solar panels. Meaning, panels that do not track the sun across the sky, a single axis solar panels allow for a better output from the photovoltaic cells. To collect the data from the solar panel like voltage, current, power etc., needs manpower and the job is tedious using the Internet of Things Technology for supervising solar photovoltaic, monitoring and maintenance of the plant.

\subsection{Problem Objectives}

To construct a model prototype of solar panel movement system with a mechanical assemble to move the panel East to West 180 degree. Globally access the information like solar panel voltage, battery voltage, charging current, via internet on smart phones from anywhere in the world using IOT (internet of things)[8]. To prevent the battery from over-charging, deep discharge and provide constant charging voltage and current. To constant a DC to AC inverter hardware circuit to operate an AC load of $15 \mathrm{~W}$ from the charged battery

\subsection{Methodology Adopted}

a) 32-bit Microcontroller is used as a real time digital monitoring and control system.

b) BLYNK Google play application for globally accessing of information.

c) H-bridge for DC motor ARM direction control.

d) DC to DC buck converter to provide constant charging voltage and current to charge battery more effectively.

e) DC to AC converter is used to convert DC $12 \mathrm{~V}$ to AC $220 \mathrm{~V}$.

\subsection{Specification}

\section{Hardware Requirement}

a) $10 \mathrm{~W}$ solar panel

b) 12 volts rechargeable battery

c) Parallel inverter circuit

d) ESP8266 (Wi-Fi adapter)

e) L2393D H-bridge driver

f) LDR's

g) $10 \mathrm{rpm}$ low speed high torque geared DC motor

h) AT91SAM3XBE Microcontroller (32bit RISC controller)

\section{Software Requirement}

a) Sketch-IDE (Integrated development environment) 1.8 .0 or higher.

b) BLYNK- application or app for accessing information on smart phone.

\section{II.BLOCK DIAGRAM}

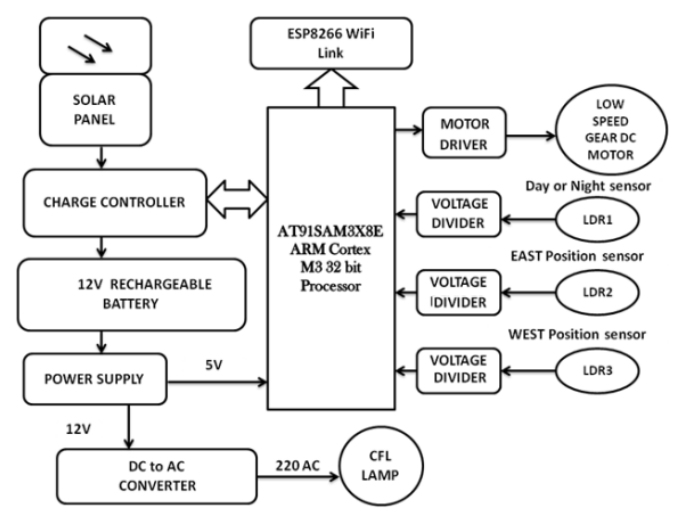

Figure 2.1: Block Diagram of Design and Fabrication of Single axis Solar and IoT Based Monitoring System.

\subsection{Solar Panel}

The photovoltaic module, known more commonly as the solar panel, is used as a component in a larger photovoltaic system to offer electricity for commercial and residential applications. Because a single photovoltaic module can only produce a limited amount of power, many installations contain several modules or panels and this is known as a photovoltaic array[11] is as shown in figure 2.1.

\subsection{AT91SAM3X8E (Microcontroller)}

AT91SAM3X8E is based on the ARMCortexM3 32bit processor, the Atmel SAM3X8E runs at $84 \mathrm{MHz}$ and features $512 \mathrm{~KB}$ of flash memory in $2 \times 256 \mathrm{~KB}$ banks and $100 \mathrm{~KB}$ of SRAM in $64 \mathrm{~KB}+32 \mathrm{~KB}$ banks, with an additional $4 \mathrm{~KB}$ as NFC (NAND Flash controller) SRAM. 
The SAM3X8E also features a 12-bit ADC/DAC, temperature sensor, 32-bit timers, PWM timer and RTC. The 16-bit external bus interface supports SRAM, PSRAM, NOR and NAND Flash with error code correction [6]. The device operates from $1.62 \mathrm{~V}$ to $3.6 \mathrm{~V}$ and is available in 144-pin packages.

\subsection{Low Speed Gear Dc Motor}

A unit which creates mechanical energy from electrical energy, and which transmits mechanical energy through the gearbox at a reduced speed as shown in figure 2.2. A gear head and motor combination to reduce the speed of the motor to obtain the desired speed or torque.

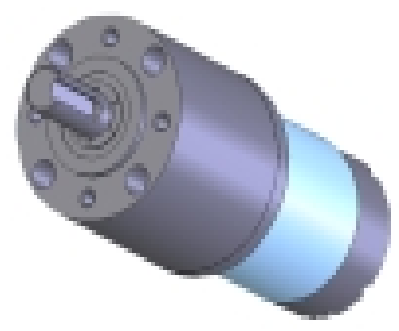

Figure 2.2: Low Speed Gear DC motor

A DC servomotor has an output shaft that can be positioned by sending a coded signal to the motor. As the input to the motor changes, the angular position of the output shaft changes as well. Servomotors are generally small and powerful for their size, and easy to control. Common types of DC servomotors include brushless or gear motor types.

\subsection{Light Sensor / Photo Detector}

A photo resistor or Light-Dependent Resistor (LDR) or photocell is a resistor whose resistance decreases with increasing incident light intensity; in other words, it exhibits photoconductivity as shown in figure2.3.
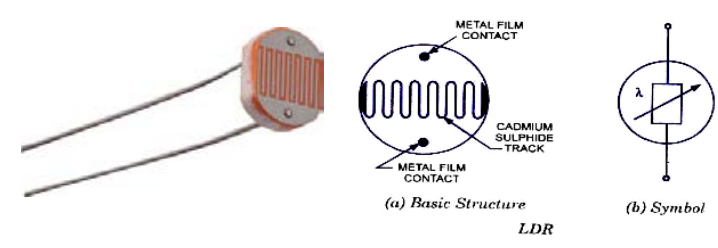

Figure 2.3: Light Sensor / Photo Detector (symbol \& basic structure)
A photo resistor is made of a high resistance semiconductor. If light falling on the device is of high enough frequency, photons absorbed by the semiconductor give bound electrons enough energy to jump into the conduction band. The resulting free electron (and its hole partner) conduct electricity, thereby lowering resistance.

\subsection{Voltage Regulator}

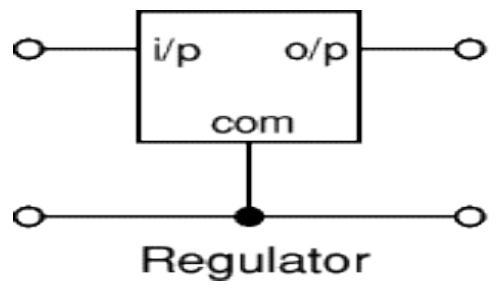

Figure 2.4: 7805 3-Terminal 1A Positive voltage Regulators

Voltage regulator ICs are available with fixed (typically 5V, 6V, 9V, $12 \mathrm{~V}$ etc.,) or variable output voltages as shown in figure 2.4.

The LM78XX series of three-terminal positive regulators are available in the TO-220/D-PAK package and with several fixed output voltages, making them useful in a wide range of applications. Each type employs internal current limiting, thermal shut-down and safe area protection, making it essentially indestructible. If adequate heat sinking is provided, they can deliver over 1A output current. Although designed primarily as fixed voltage regulators, these devices can be used with external components to obtain adjustable voltages and currents.

\subsection{Driver Circuit}

A Darlington pair is two transistors that act as a single transistor but with a much higher current gain as shown figure 2.5. Transistors have a characteristic called current gain. This is referred to as its hFE. The amount of current that can pass through the load when connected to a transistor that is turned on equals the input current $\mathrm{x}$ the gain of the transistor (hFE).The current gain varies for different transistor and typically it may be 100 . This would mean that the current available to drive the load would be 100 times larger than the input to the transistor. 


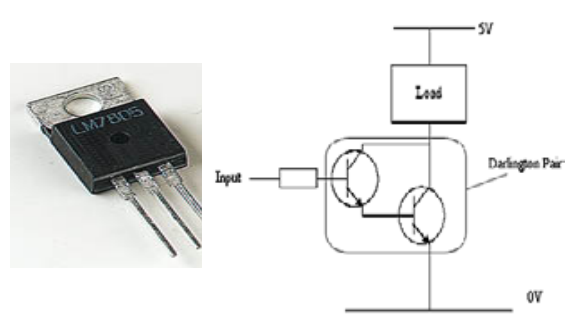

Figure 2.5: Driver Circuit

\section{7: 12v Rechargeable Battery}

The lead-acid battery was invented in 1859 by French physicist Gaston Planteand is the oldest type ofrechargeable battery.Despite having a very low energy-to-weight ratio and a low energy-to-volume ratio, its ability to supply high surge currents means that the cells have a relatively largepower-to-weight ratio. These features, along with their low cost, make it attractive for use in motor vehicles to provide the high current required byautomobile starter motors.Lead-acid batteries are widely used even when surge current is not important and other designs could provide higher energy densities. Lead-acid are widely used for storage in backup power supplies in cell phone towers, highavailability settings like hospitals, andstandalone power systems. For these roles, modified versions of the standard cell may be used to improve storage times and reduce maintenance requirements.

\section{III.AURDINO TECHNOLOGY}

Arduino is a tool for making computers that can sense and control more of the physical world than your desktop computer. It's an open-source physical computing platform based on a simple microcontroller board, and a development environment for writing software for the board as shown in figure 3.1.Arduino simplifies the process of working with microcontrollers, but it offers some advantage for teachers, students, and interested amateurs over other systems:

a) Inexpensive - Arduino boards are relatively inexpensive compared to other microcontroller platforms.

b) Cross-platform - The Arduino software runs on Windows, Macintosh OSX, and Linux operating systems. Most microcontroller systems are limited to Windows.

c) Simple, clear programming environment - The Arduino programming environment is easy-to-use for beginners, yet flexible enough for advanced users to take advantage of as well.

d) Open source and extensible softwareThe Arduino software is published as open

e) source tools, available for extension by experienced programmers

f) The language can be expanded through $\mathrm{C}++$ libraries, and people wanting to understand the technical details can make the leap from Arduino to the AVR $\mathrm{C}$ programming language on which it's based. Open source and extensible hardware - The Arduino is based on Atmel's ATMEGA8 and ATMEGA168 microcontrollers. Even relatively inexperienced users can build the breadboard version of the module in order to understand how it works and save money

\subsection{Arduino Due (Development Board)}

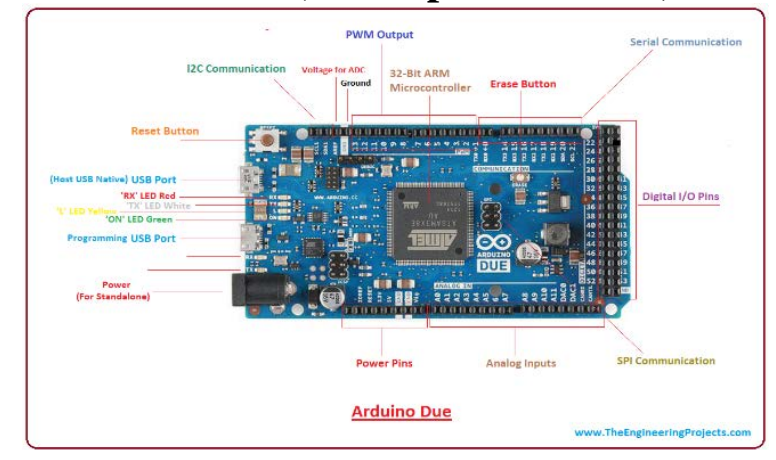

Figure 3.1: Arduino due (Development board) ,Courtesy Guihua.Li ,2012

The Arduino Due is a microcontroller development board based on the Atmel SAM3X8E ARM Cortex-M3 CPU AT91SAM3X8E. It is a development board based on a 32-bit ARM core microcontroller. It has 54 digital input/output pins (of which 12 can be used as PWM outputs), 12 analog inputs, 4 UARTs (hardware serial ports), a $84 \mathrm{MHz}$ clock, an USB OTG capable connection, 2 DAC (digital to analog), 2 TWI, a power jack, an SPI header, a JTAG header, a reset button and an erase button. The board contains everything needed to support the microcontroller, simply connect it to a computer with a micro-USB cable or power it with a ACto-DC adapter or battery to get started. 
The Arduino Due can be powered via the USB connector or with an external power supply. The power source is selected automatically. External (non-USB) power can come either from an AC-to-DC adapter (wallwart) or battery.

\section{IMPLEMENTATION}

\subsection{Hardware Implementation}

The working of arudino due is $3.3 \mathrm{~V}$ which is supplied by the voltage regulator as shown in figure 4.1. The circuit board as two relays(electromechanical) that is high cut off relay which is used for battery for greater than $14 \mathrm{~V}$ (i.e., voltage greater than $14 \mathrm{~V}$ will be cut off) and low cut off relay which is used for load than $11 \mathrm{~V}$ (i.e, voltage less than $11 \mathrm{~V}$ will be neglected), relay is normally in closed position and connected to battery as shown in figure 4.3 .

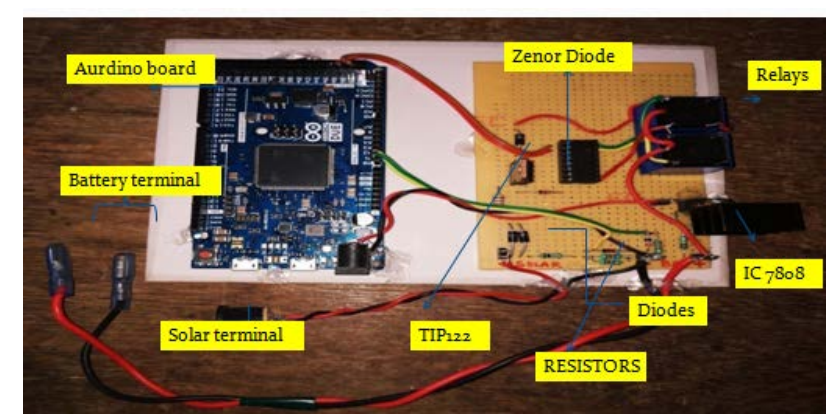

Figure 4.1: Figure shows the single axis solar tracker

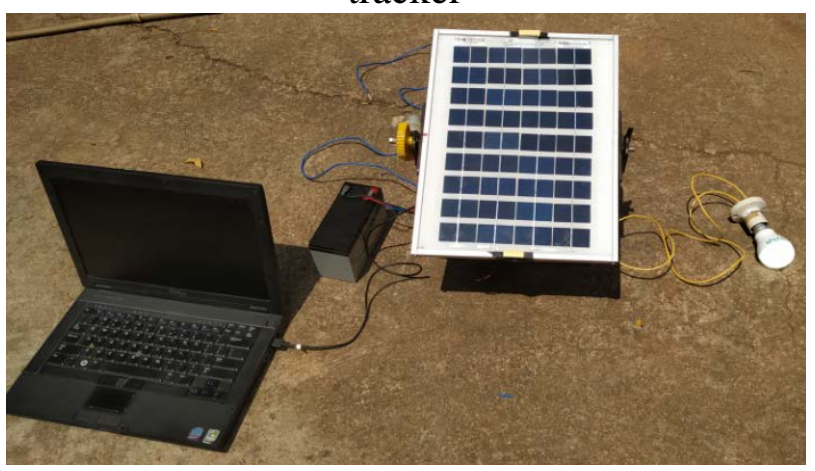

Figure 4.2: Implementation of solar tracking on single axis

To operate the relay a driver circuit(ULN2003).It is a darlington emitter follower,it as 7 input and 7 output pins. A diode which is used forward bias which allows the voltage from solar panel to battery(unidirectional).A motor with the speed of 2520rpm is used. The speed is high and torque is low, to avoid this a gear box is used which reduces the speed from 2520rpm to 3.5rmp and torque is increased. The shaft of the motor is directly connected to a gear known as "Worm Gear".An H-bridge(L239D) is used to make the motor rotate clockwise,anti-clockwise and remain halt position.

ESP8266 wifi adapter which works at $2.4 \mathrm{GHz}$ of carrier frequency and baud rate of 1,15,200 is present which requires $3.3 \mathrm{~V}$ which is supplied by the regulator AMS1117. A dc-dc buck converter (LM2596-IC) which is a variable power supplyand can deliver upto 3A of current is given to the LDR's.

Protocol of communication is UART with baud rate of 1,15,200. A step-up transformer,an oscillator circuit of frequency $50 \mathrm{hz}$ is given to the MOSFET's which operates alternatively a capacitor is connected parallel across the transformer to remove noise or ripples generated. An output of $220 \mathrm{~V}$ is produced which can be given to the load.

\subsection{Software Implementation}

The Sketch IDE (Integrated Development Environment) is a special program running on your computer that allows you to write sketches for the Arduino board in a simple language modelled after the Processing language as shown in figure 4.3. The magic happens when you press the button that uploads the sketch to the board: the code that you have written is translated into the $\mathrm{C}$ language, and is passed to the AVR-GCC compiler, an important piece of open source software that makes the final translation into the language understood by the microcontroller. This last step is quite important, because it's where Arduino makes your life simple by hiding away as much as possible of the complexities of programming microcontrollers.

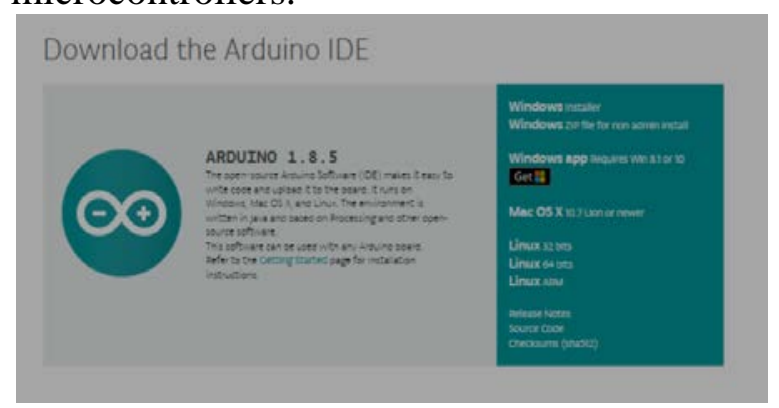

Figure 4.3: Figure shows the view of Sketch program 


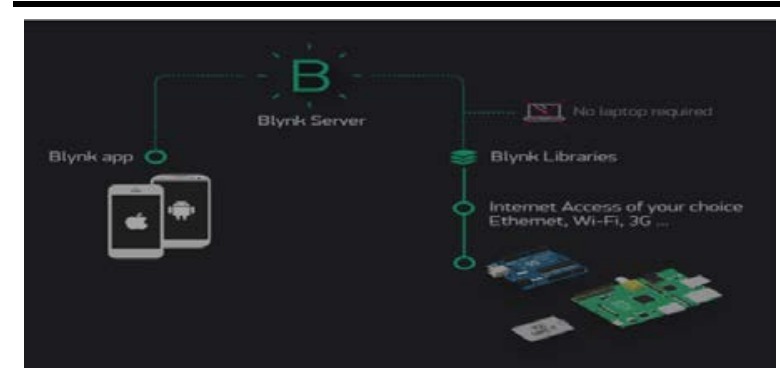

Figure 4.4: Figure shows the Blynk App

The programming cycle on Arduino is basically as follows:

$\checkmark$ Plug your board into a USB port on your computer.

$\checkmark$ Write a sketch that will bring the board to life.

$\checkmark$ Upload this sketch to the board through the USB connection and wait a couple of seconds for the board to restart.

$\checkmark$ The board executes the sketch that you wrote.

The idea of sketching in code is a way of thinking about writing code as a simple intuitive process, just like drawing in a sketchbook. In this way, an Arduino program is called a sketch and is saved in a folder called a sketchbook. Sketching means we can get our hands dirty and quickly try out a new idea. It is a skill available to all of us.

\section{Blynk}

Blynk is designed for the Internet of Things. It can control hardware remotely, it can display sensor data as shown in figure 4.4, it can store data, visualize it and do many other cool things.

There are three major components in the platform:

- Blynk App-allows to you create amazing interfaces for your projects using various widgets we provide.

- Blynk Server - responsible for all the communications between the smartphone and hardware. You can use our Blynk Cloud or run your private Blynk server locally. Its open-source, could easily handle thousands of devices and can even be launched on a Raspberry Pi.

- Blynk Libraries - for all the popular hardware platforms - enable communication with the server and process all the incoming and outcoming commands.
Blynk works over the Internetthis means that the hardware you choose should be able to connect to the internet.After you download the Blynk App, you'll need to create a New Blynk account. This account is separate from the accounts used for the Blynk Forums, in case you already have one. After you've successfully logged into your account, start by creating a new project. Select the hardware model you will use.Auth Token is a unique identifier which is needed to connect your hardware to your smartphone. Every new project you create will have its own Auth Token.

You'll get Auth Token automatically on your email after project creation. You can also copy it manually. When you are done with the Settings - press the playbutton. This will switch you from EDIT mode to PLAY mode where you can interact with the hardware. While in PLAY mode, you won't be able to drag or set up new widgets, press stop and get back to EDIT mode.

You will get a message saying "Arduino MEGA is offline". We'll deal with that in the next section.

\section{RESULTS AND ANALYSIS}

The results for the project were got from LDRs for the solar tracking system. The results were recorded and tabulated. The outputs of the LDRs were dependent on the light intensity falling on their surfaces. Arduino has a serial that communicates on digital pins RX and TX as well as with the computer through a USB. Arduinoenvironment's built in serial monitor can be used to communicate with the aurdino board. To collect the results figure shown in 5.1 with solar tracker and figure shown in 5.2 without solar tracker, a code was written that made it possible to collect data from the LDRs after every half an hour. By measuring the light intensity at a given time it will be possible to get the difference in efficiency between solar tracking and without solar tracking. The results were obtained. The values obtained were recorded and used to draw graphs to show the relations. 


\subsection{Without Solar tracker}

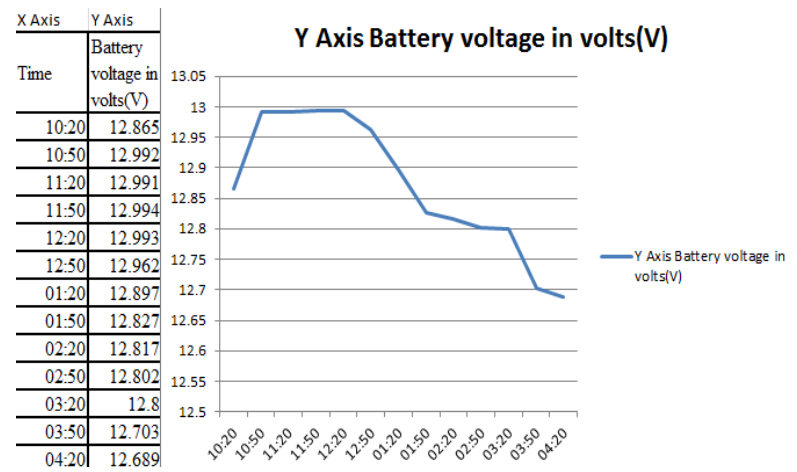

Figure 5.1:Without tracker battery voltage v/s time

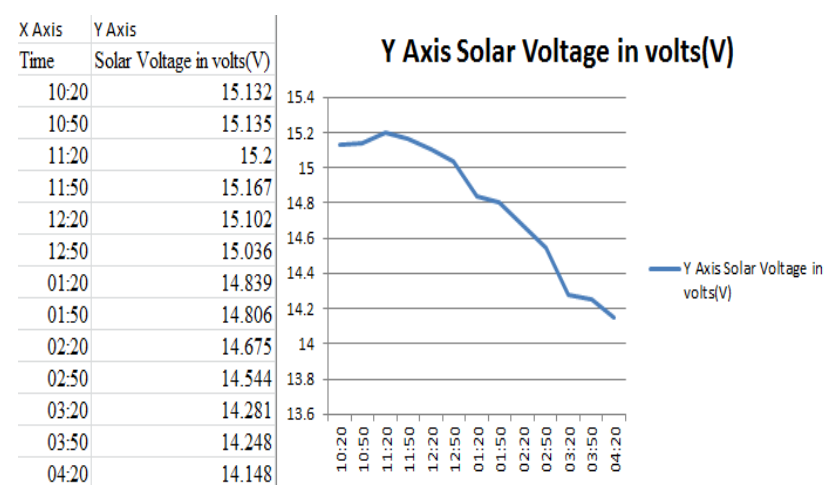

Figure 5.2: Without tracker solar voltage v/s time

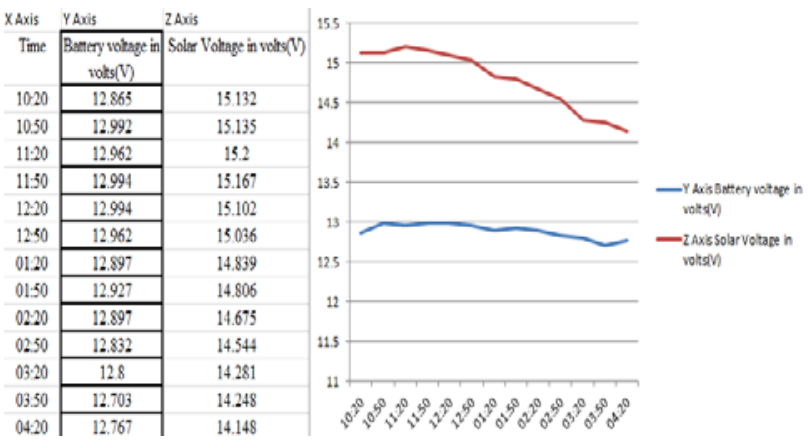

Figure 5.3: Without tracker battery and solar voltage v/s time

\subsection{WithSolar tracker}

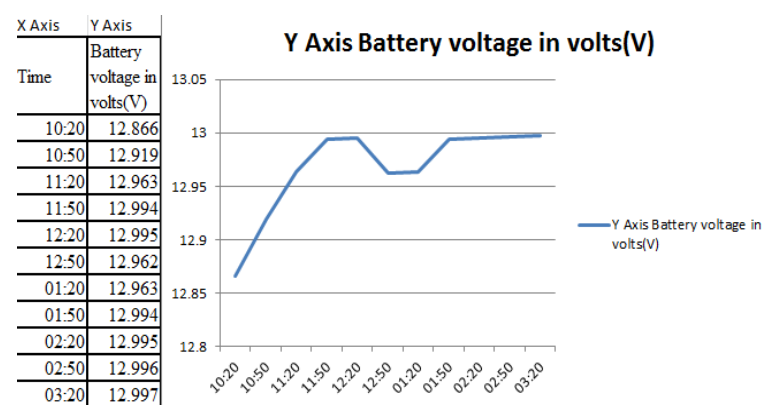

Figure 5.4: With tracker battery voltage v/stime

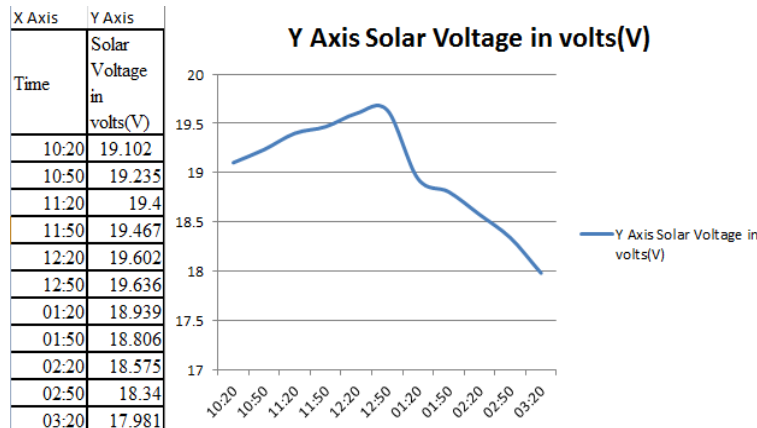

Figure 5.5:With tracker solar voltage v/s time

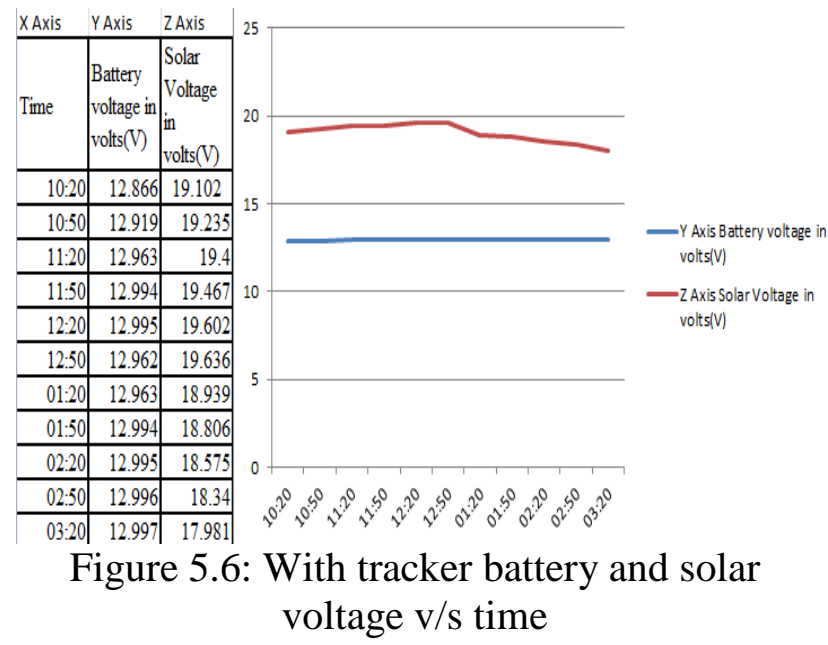

\section{CONCLUSION AND FUTURE SCOPE}

\subsection{Advantages}

a) Solar trackers generate more electricity than stationary counter parts due to an increased direct exposure to solar rays.

b) Generally a lower cost than dual-axis trackers.

c) Higher reliability than dual-axis trackers.

d) Higher life span than dual-axis trackers.

e) Single axis trackers tend to be better for companies with a lower budget or for areas with frequent cloud cover

\subsection{Disadvantages}

a) Lower energy output during sunny conditions when compared to dual axis trackers

b) Fewer technology advancements

c) Single axis trackers do not allow for two degrees of flexibility

\subsection{Applications}

It is used for
a) Agricultural purpose
b) Generate electricity
c) Residential \& Commercial purpose
d) Traffic lights
e) Road reflectors
f) Solar heaters 


\subsection{Conclusion}

A solar panel that tracks the sun was designed and implemented. The required program was written that specified the various actions required for the project to work. As a result tracking was achieved. After examining the information obtained in the data analysis section it can be said that the proposed sun tracking solar array system is a feasible method of maximizing the energy received from solar radiation. The system designed was a single axis tracker. The use of stepper motors enables accurate tracking of the sun while keeping track of the array's current position in relation to its initial position. The automatic solar tracker is an efficient system for solar energy collection. It has been shown that the sun tracking systems can collect about 20\% more energy than a fixed panel system. Thus high efficiency is achieved through this tracker. This project was implemented with minimum resources. The circuitry was kept simple, while ensuring efficiency is not affected.

\subsection{Future Scope}

It has been proven through our research and statistical analysis that solar tracking system with single axis tracker can increase energy output by approximately $40.6 \%$.

Further mechanical enhancement can be done to the prototype, to implement dual-axis tracking

\section{Bibliography}

[1] O. Aliman, and I Daut, "RotationElevation of Sun Tracking Mode to Gain High Concentration Solar Energy”, The IEEE International Conference on Power Engineering, Energy and Electrical Drives, pp.551-555, 2007.

[2] R. Singh, and Y.R. Sood, "Transmission tariff for restructured Indian power sector with special consideration to promotion of renewableenergy sources”, The IEEE Conference TENCON-2009, pp. 1-7, 2009.

[3] Kassem (IEEE Member) and M. Hamad "A Microcontroller-Based Multi-Function Solar Tracking System” IEEE Conference 978-14244-9493-4/11/\$26.00,2011.

[4] Md. Ismail Hossain, Shakil Ahamed Khan, Md. Shafiullah, "Power Maximization of a Photovoltaic System Using Automatic Solar Panel Tracking along with Boost Converter and Charge Controller" IEEE Conference 978-14673-1436-7/12/\$31.00,2012.

[5] Arslan A. Rizvi, Khaled Addoweesh "Sun Position Algorithm for Sun Tracking Applications" IEEE Conference 978-1-47994032-5/14/\$31.00,2014.

[6] ErsanKabalc1, Yasin KABALCI Nigde, A Single-Axis Solar Tracking System and Monitoring Software"IEEE Conference 978-14673-6647-2/15/\$31.00, 2015

[7] SumantMalav, Shelly Vadhera, "Hardware Implementation of Solar Tracking System Using a Stepper Motor” 978-1-46786503-1/15/\$31.00,2015.

[8] J. Arai, K. Iba, T. Funabashi; Y. Nakanishi, K. Koyanagi, and R. Yokoyama, "Power electronics and its applications to renewable energy in Japan, ” The IEEE Circuits and Systems Magazine, Vol. 8, No. 3, pp.52-66, 2008.

[9] S. Armstrong and W.G Hurley "Investigating the Effectiveness of Maximum Power Point Tracking for a Solar System”, The IEEE Conference on Power Electronics Specialists, pp.204-209, 2005.

[10] N.Othman, M.I.A.Manan, Z.Othman,S.A.M.A Al Junid, “ Performance Analysis of Dual-Axis Solar Tracking System”. IEEE International Conference on Control system, Computing and Engineering, 29 Nov.-1 Dec.2013. 